\title{
Therapeutic Assessment of Primaquine for Radical Cure of Plasmodium vivax Malaria at Primary and Tertiary Care Centres in Southwestern India
}

\author{
Rishikesh Kumar', Vasudeva Guddattu², Kavitha Saravu1,3,* \\ ${ }^{1}$ Department of Medicine, Kasturba Medical College, ${ }^{2}$ Department of Statistics, ${ }^{3}$ Manipal McGill Center for Infectious Diseases, Manipal University, \\ Madhav Nagar, Manipal, Karnataka, 576104, India
}

\begin{abstract}
Acquaintance is scanty on primaquine (PQ) efficacy and Plasmodium vivax recurrence in Udupi district, Karnataka, India. We assessed the efficacy of 14 days $P Q$ regimen $(0.25 \mathrm{mg} / \mathrm{kg} /$ day $)$ to prevent $P$. vivax recurrence. Microscopically, aparasitemic adults ( $\geq 18$ years) after acute vivax malaria on day 28 were re-enrolled into 15 months' long follow-up study. A peripheral blood smear examination was performed with participants at every 1-2 month interval. A nested PCR test was performed to confirm the mono-infection with $P$. vivax. Of 114 participants, 28 (24.6\%) recurred subsequently. The median (IQR) duration of the first recurrence was 3.1 (2.2-5.8) months which ranged from 1.2 to 15.1 months, including initial 28 days. Participants with history of vivax malaria had significantly higher risk of recurrence, with hazard ratio $(\mathrm{HR})(95 \% \mathrm{Cl})$ of $2.62(1.24-5.54)(P=0.012)$. Severity of disease $(11.4 \%, 13 / 114)$ was not associated $(P=1.00)$ with recurrence. Of 28 recurrence cases, the $\mathrm{nPCR}$ proved that $P$. vivax mono-infection recurrence rate was at least $72.7 \%(16 / 22)$ at first recurrence. In Udupi district, $P Q$ dose of $0.25 \mathrm{mg} / \mathrm{kg} /$ day over 14 days seems inadequate to prevent recurrence in substantial proportion of vivax malaria. Patients with a history of vivax malaria are at high risk of recurrences.
\end{abstract}

Key words: Plasmodium vivax, malaria, anti-malarial, treatment failure, relapse, primaquine

\section{INTRODUCTION}

Of the 5 Plasmodium spp. malariogenic to humans, P. vivax and $P$. ovale have distinction to cause 'relapse'. The relapse feature is indeed one of the trickiest hurdles to be outmanoeuvred to control/eliminate the P. vivax malaria that asserts the widest global geographic range and utmost burden in Southeast Asia [1]. Primaquine (PQ) is the sole antimalarial in clinical practice for the radical cure of $P$. vivax and $P$. ovale malaria worldwide. Although P. vivax resistance to PQ has never been reported, a relapse is believed to occur due to suboptimal cumulative dosage or impaired pharmacokinetics of PQ [2]. Tailoring PQ regimen is a function of body weight, glucose6-phosphate dehydrogenase (G6PD) activity, and natural relapse rates in a given population. It is vital to measure the natural relapse prevalence in $P$. vivax malaria in a geographic region. It is said that a relapse prevalence over 50\% would ac-

- Received 5 July 2016, revised 26 August 2016, accepted 27 September 2016.

*Corresponding author (kavithasaravu@gmail.com)

(c) 2016, Korean Society for Parasitology and Tropical Medicine

This is an Open Access article distributed under the terms of the Creative Commons Attribution Non-Commercial License (http://creativecommons.org/licenses/by-nc/4.0) which permits unrestricted non-commercial use, distribution, and reproduction in any medium, provided the original work is properly cited. count for majority of vivax malaria burden and serve as prime reservoir [3].

P. vivax comprises $~ 50 \%$ malaria burden in India [4] and the national guideline recommends a PQ dosage of $0.25 \mathrm{mg} /$ $\mathrm{kg}$ body weight daily over 14 days in all non-pregnant adult vivax malaria patients with normal G6PD activity [5]. Chloroquine (CQ) and artemisinin combination drugs remain the companion antimalarials to PQ for uncomplicated and complicated vivax malaria, respectively [5]. There have been reports describing P. vivax relapse from different parts of India [6-10]; however, there lacks one such from Udupi district, which remains one of the most fiercely active malaria transmission zones of Karnataka, India, having annual parasite incidence (API) of $>2$ per 1,000 risk population and about $90 \%$ P. vivax prevalence. Also, some previous reports indicated low relapse rates $[11,12]$ which appear to be underestimated due to a short follow-up length. We undertook this study to determine the efficacy of PQ ( $0.25 \mathrm{mg} / \mathrm{kg}$ body weight daily over 14 days) for preventing recurrences of $P$. vivax across 15 months duration, including participants from a tertiary and 5 primary health centres of Udupi taluk in Karnataka, India. 


\section{MATERIALS AND METHODS}

\section{Study design and population}

Light microscopically proven $P$. vivax mono-infected adults ( $\geq 18$ years) from 1 tertiary care hospital, Kasturba Hospital (KH), Manipal, Udupi, and 5 primary health centres (PHCs) of Udupi taluk (Hiriadka, Kolalagiri, Malpe, Pethri, and Shirva), pre-enrolled in a CQ-PQ combined therapeutic trial $[13,14]$, upon convalescence on 28th day were requested to participate in another 15 months long follow-up study. All participants had 'adequate clinical and parasitological response' and were microscopically aparasitemic at recruitment on the day 28. All voluntarily consenting participants were recruited from September 2012 through October 2014. Participants were followed to check parasitemia at every 1-2 month's duration and additionally if anyone had fever. All participants from PHCs and 125 participants from $\mathrm{KH}$, Manipal were treated previously with CQ $25 \mathrm{mg} / \mathrm{kg}$ body weight over 3 days and PQ $0.25 \mathrm{mg} / \mathrm{kg}$ body weight daily for 14 days. Patients with G6PD deficiency, i.e., activity less than 30\% of the normal mean [15], who could not be treated with PQ, $0.25 \mathrm{mg} /$ $\mathrm{kg}$ body weight daily for 14 days, were excluded. Adherence to the prescribed CQ-PQ was not observed directly, rather on each follow-up till day 28, participants were reminded to comply with the regimen and emptied drug stripes were documented. Besides, 37 out of total 162 patients at KH, Manipal were treated with artesunate combination therapy (ACT) along with PQ as per clinicians' decision (Fig. 1).

\section{Ethics}

Ethical approval (no. IEC 86/2013) for the study was obtained from the institutional ethics committee of Kasturba Medical College and Kasturba Hospital, Manipal University, Manipal, Karnataka, India. A written informed consent was obtained from all participants before their enrolment into the study.

\section{Study setting}

Fig. 2 depicts the geographical location of the KH, Manipal and the PHCs. Udupi district harbours tropical monsoon climate and over 4,000 mm rainfall is recorded per annum during the month of June to October. The malaria incidence occurs throughout a year in Udupi taluk, and the mean annual parasite incidence was estimated as 2.5 per 1,000 risk population during the year 2012 to 2014. The catchment area of the

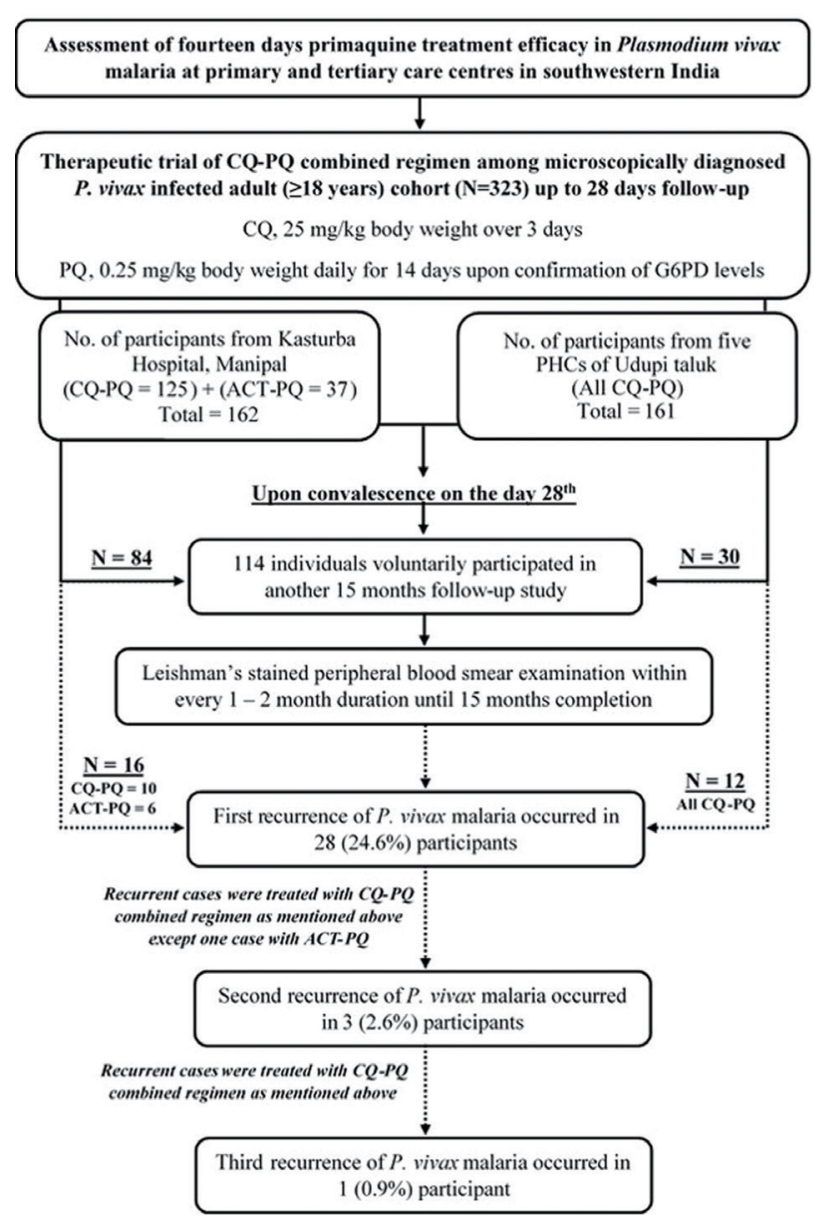

Fig. 1. Flow diagram depicting the study protocol and outcome.

KH, Manipal encompasses both rural and urban population of coastal and interior Karnataka, Goa, and Kerala. The KH, Manipal records malaria incidence across the year with over 200 malaria cases per annum, comprising > 55\% P. vivax cases. By contrast, the catchment areas of the 5 PHCs are largely rural, except Hiriadka PHC wherein rapid urbanization and megaconstruction projects are in-progress, mainly around Manipal.

\section{Blood sample collection and processing}

Both thin and thick peripheral blood smears were obtained by capillary puncture from every participant on each followup. Smears were stained using Leishman's stain, then examined individually by 3 microscopists to ascertain $P$. vivax mono-infection and to determine the parasite index (PI) per $\mu \mathrm{l}$ blood [16]. On every instance of $P$. vivax reappearance, another $1 \mathrm{ml}$ venous blood was obtained from participants in a $\mathrm{K}_{2} \mathrm{ED}$ TA anticoagulated vacutainer for total leukocyte count and DNA extraction. Participants' total leukocyte count per $\mu$ l was 


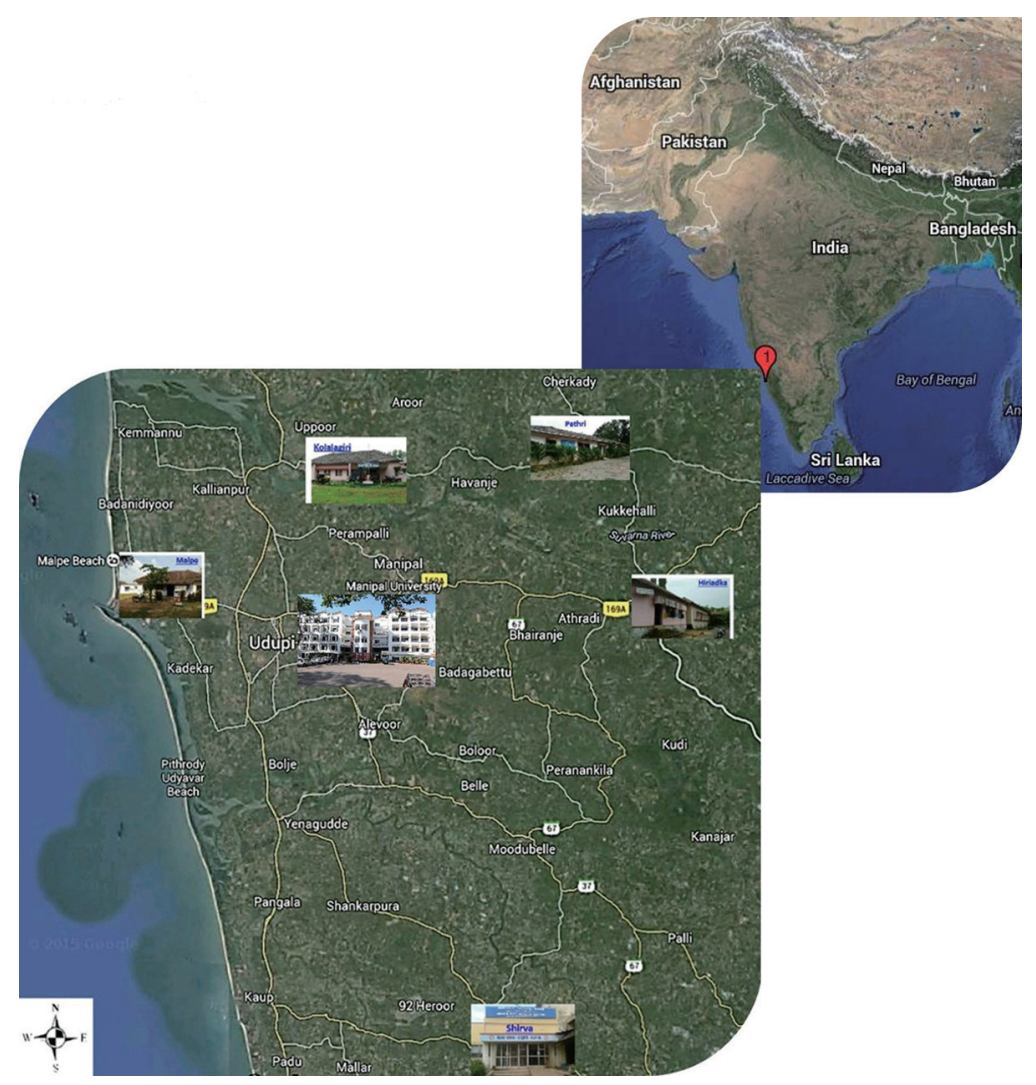

Fig. 2. Geographical locations of Kasturba Hospital, Manipal and primary health centres of Hiriadka, Kolalagiri, Malpe, Pethri and Shirva of Udupi taluk, Karnataka, India. Image adapted from USGS Earth Resources Observatory and Science (EROS) Center (public domain): http://earthexplorer.usgs.gov/.

estimated by Beckman Coulter ${ }^{\circledR}$ LH 750 Hematology Analyzer (Fullerton, California, USA) and used for PI calculation. Further, DNA extraction was carried out from $200 \mu$ of $K_{2}$ EDTA anticoagulated blood using QIAamp DNA Blood Mini Kit (Hilden, Germany) as per the manufacturer's instructions and stored at $-20^{\circ} \mathrm{C}$ until the conduct of nested PCR (nPCR) test for confirmation of the mono-infection with P. vivax malaria.

\section{Exposure variables}

Axillary temperature and history of fever within last 1 week was recorded on each follow-up. Pertinent clinical and laboratory variables were assessed and recorded in a proforma.

\section{Outcome variables}

Anytime during the study period, reappearance of $P$. vivax parasitemia as confirmed by light microscopy was considered the primary outcome. All reappearances were considered 'recurrence' as molecular investigations to differentiate cases into either 'relapse' or 'reinfection' was not performed. Demo- graphic, clinical, and laboratory variables were compared between 'no-recurrence' and 'recurrence' groups to determine mutual associations. Severity of $P$. vivax malaria was the secondary outcome. Severity was determined as per the WHO's guideline for management of severe malaria [17].

\section{Patients' treatment}

Specific anti-malarial medications were prescribed to recurrent cases as per the clinicians' decision and the national guideline for treatment of malaria [5]. CQ-PQ was the most frequently prescribed anti-malarial regimen. However, ACT, i.e., artesunate plus doxycycline, along with PQ was given to cases befitting so as per the attending clinicians. Activity of G6PD enzyme (U/g hemoglobin) was estimated by spectrophotometric kinetic 'gold standard' method.

\section{CQ-PQ regimen at Kasturba Hospital, Manipal}

Resochin (chloroquine phosphate $250 \mathrm{mg}$; CQ, $25 \mathrm{mg} / \mathrm{kg}$ body weight over 3 days) manufactured by Bayer Pharmaceu- 
ticals Private Ltd. (Bayer House Central Avenue, Maharashtra, India) and Malirid (primaquine phosphate $7.5 \mathrm{mg}$; PQ, 0.25 $\mathrm{mg} / \mathrm{kg}$ body weight daily over 14 days) manufactured by IPCA Laboratories Pvt. Ltd. (Mumbai, Maharashtra, India) was administered to the study cohort. Notably, PQ was administered only after estimation of G6PD activity, but immediately after completion of CQ dosage.

\section{$\mathrm{CQ}-\mathrm{PQ}$ regimen at $\mathrm{PHCs}$, Udupi taluk}

CQ $(25 \mathrm{mg} / \mathrm{kg}$ body weight over 3 days $)$ and PQ $(0.25 \mathrm{mg} /$ kg body weight daily over 14 days) as supplied and procured by the National Vector Borne Disease Control Programme (NVBDCP), India were dispensed among participants by the staffs of respective PHCs as per the national guideline [5]. CQ and PQ were started together before the estimation of G6PD activity. However, G6PD results were available by $48 \mathrm{hr}$ after the onset of CQ-PQ regimen.

\section{Confirmation of Plasmodium vivax mono-infection by nPCR test}

Small sub-unit ribosomal RNA was amplified using genus and species-specific oligonucleotide primers separately for $P$. vivax and $P$. falciparum [18] with modifications in the amplification conditions of the second step [13]. DNA extracted from the reference samples procured from the National Institute of Malaria Research (ICMR), Dwarka, Delhi-110077, India served as positive controls in every batch of nPCR tests. Whereas, DNA extracted from a healthy volunteer was used as negative control.

\section{Statistical analyses}

Continuous variables were summarized as mean with SD or median with interquartile range (IQR). Categorical variables were summarized as frequency with proportion. No-recurrence and recurrence groups were compared by independent sample $t$-test or Mann Whitney U test and chi-square test or Fischer's exact test. Furthermore, variables of recurrence cohort at initial presentation and at first recurrence were compared using paired $t$-test or Willcoxon sign rank test and McNemar chi-square test. All tests of significance were 2-tailed with a $P$ value $<0.05$ indicating statistical significance. Parasite index was summarized as geometric mean with $95 \%$ confidence interval (CI). Cox regression analysis was performed to determine the hazard ratio (HR) of history of P. vivax malaria for recurrence. Furthermore, a Kaplan-Meier survival plot was gen- erated to determine the survival function of recurrences till 15 months follow-up duration for independent variables. Log rank test was used to compare the survival function of independent variables. Data analysis was done using Statistical Package for the Social Sciences version 15.0 (SPSS, South Asia, Bangalore, India).

\section{RESULTS}

\section{Baseline characteristics of the study cohort}

Of total 323 participants in 28 days CQ-PQ therapeutic trial, 114 participated in 15 months follow-up study (Fig. 1). The study cohort included 105 (92.1\%) male and 9 (7.9\%) female participants. The mean $( \pm$ SD) age of cohort was $38.9( \pm 12.4)$ years which ranged from 18 to 76 years. The mean $( \pm S D)$ G6PD enzyme activity of study cohort was $14.5 \pm 3.8 \mathrm{U} / \mathrm{g}$ hemoglobin ranging from 5.5 to $27.2 \mathrm{U} / \mathrm{g}$ hemoglobin. No PQ related adverse effects were noted among the study cohort. All cases with subsequent recurrence were retreated using CQ-PQ combined regimen as described above except 1 case that was treated with ACT-PQ at first recurrence (Fig. 1).

\section{Study outcomes}

Of 114 participants, 28 (24.6\%) recurred subsequently, including 2 participants with 2 recurrences and 1 participant with 3 recurrences through 16 months (since the date of recruitment in the 28 days CQ-PQ combined efficacy trial). One patient did present with $P$. falciparum malaria after 3 months of initial presentation. Cumulative proportion of recurrence across 15 months was significantly higher $(P=0.03)$ in PHCs' cohort than KH, Manipal cohort (Fig. 3A). Through 16 months of follow-up, the median (IQR) duration of first recurrence was 3.1 (2.2-5.8) months and ranged from 1.2 to 15.1 months. Sub-groups treated with antimalarial regimens viz. CQ-PQ and ACT-PQ at KH, Manipal did not show any significant $(P=0.08)$ difference in their respective recurrence proportions (Table 1).

Recurrence proportion did not vary by age, gender, clinical symptoms, and vital signs on presentation (Table 1). Except total leucocyte count and serum alanine aminotransferase, there was no difference observed in the laboratory profile between recurrence and no-recurrence groups (Table 2). In recurrence group, proportion of cases managed on ambulatory care rose significantly $(P=0.02)$ at first recurrence than initial presentation (Table 3 ). In recurrence group, none of the variables 
Table 1. Comparison of demographic and clinical variables between no-recurrence and recurrence groups at initial presentation

\begin{tabular}{|c|c|c|c|}
\hline \multirow{2}{*}{ Variables ${ }^{a}$} & \multirow{2}{*}{$\begin{array}{l}\text { No-recurrence }(\mathrm{N}=86)(\%) \\
\text { Mean } \pm \mathrm{SD} \text { or median }(\mathrm{IQR})\end{array}$} & \multirow{2}{*}{$\begin{array}{c}\text { Recurrence }(\mathrm{N}=28)(\%) \\
\text { Mean } \pm \mathrm{SD} \text { or median }(\mathrm{IQR})\end{array}$} & \multirow{2}{*}{$P$-value ${ }^{a}$} \\
\hline & & & \\
\hline Settings & & & $0.03^{b}$ \\
\hline Kasturba Hospital, Manipal & $68(79.1)$ & $16(57.1)$ & \\
\hline Primary Health Centres, Udupi taluk & $18(20.9)$ & $12(42.9)$ & \\
\hline Point-of-care & & & 0.19 \\
\hline Ambulatory & $36(41.9)$ & $16(57.1)$ & \\
\hline Inpatient & $50(58.1)$ & $12(42.9)$ & \\
\hline Age in years & $38.9 \pm 12.1$ & $38.9 \pm 13.6$ & 1.00 \\
\hline Gender & & & 0.69 \\
\hline Male & $80(93)$ & 25 (89.3) & \\
\hline Female & $6(7)$ & $3(10.7)$ & \\
\hline History & & & 0.67 \\
\hline Fever in days & $4(3-5)$ & $3(2-5)$ & 1.00 \\
\hline Headache & $72(83.7)$ & $24(85.7)$ & 0.66 \\
\hline Cough & $35(40.7)$ & $13(46.4)$ & 0.51 \\
\hline Vomiting & $33(38.4)$ & $13(46.4)$ & 0.29 \\
\hline Diarrhea & $11(12.8)$ & $1(3.6)$ & 0.08 \\
\hline Malaria & $31(36)$ & $16(57.1)$ & $0.02^{\mathrm{b}}$ \\
\hline P. vivax malaria & $27(31.4)$ & $16(57.1)$ & \\
\hline Vitals & & & 0.30 \\
\hline Axillary temperature at presentation $\left({ }^{\circ} \mathrm{F}\right)$ & $100 \pm 1.5$ & $99.7 \pm 1.5$ & 0.08 \\
\hline Pulse rate (beats/minute) & $85.9 \pm 11.3$ & $91.3 \pm 10.2$ & 0.71 \\
\hline Respiratory rate (breaths/minute) & $22 \pm 3.9$ & $21.6 \pm 4.2$ & 0.14 \\
\hline Systolic blood pressure (/mmHg) & $120.9 \pm 15.5$ & $114.4 \pm 16.3$ & 0.43 \\
\hline Diastolic blood pressure (/mmHg) & $78.4 \pm 10.7$ & $76 \pm 12.3$ & \\
\hline $\mathrm{nPCR}$ diagnosis & & & 0.78 \\
\hline P. vivax mono-infection & $71(82.6)$ & $22(78.6)$ & \\
\hline Mixed $P$. vivax and $P$. falciparum & $15(17.4)$ & $6(21.4)$ & \\
\hline Severe malaria $^{c}$ & $10(11.6)$ & $3(10.7)$ & 1.00 \\
\hline Antimalarial treatment at $\mathrm{KH}$, Manipal & $N=68$ & $N=16$ & 0.08 \\
\hline$C Q-P Q$ & $57(83.8)$ & $10(62.5)$ & \\
\hline ACT-PQ & $11(16.2)$ & $6(37.5)$ & \\
\hline
\end{tabular}

${ }^{a}$ Categorical variables are summarized as frequency with proportion whereas continuous variables are summarized as either mean $( \pm$ SD) or median (IQR). $\chi^{2}$-test or Fischer's exact test and independent sample $t$-test or Mann Whitney $U$ test was performed.

${ }^{\mathrm{b} P} \mathrm{P}$-value less than 0.05 showing a statistically significant difference.

'As per the WHO's guideline for management of severe malaria in the year 2012 [17].

Table 2. Comparison of laboratory variables between no-recurrence and recurrence groups at initial presentation

\begin{tabular}{|c|c|c|c|}
\hline \multirow{2}{*}{ Variables } & \multirow{2}{*}{$\begin{array}{c}\text { No-recurrence }(\mathrm{N}=86) \\
\text { Mean } \pm \mathrm{SD} \text { or median }(\mathrm{IQR})\end{array}$} & \multirow{2}{*}{$\begin{array}{c}\text { Recurrence }(\mathrm{N}=28) \\
\text { Mean } \pm \mathrm{SD} \text { or median }(\mathrm{IQR})\end{array}$} & \multirow{2}{*}{$P$-value } \\
\hline & & & \\
\hline Total leucocyte count (cells/mm³) & $5,056 \pm 1,658$ & $5,907 \pm 1,905$ & $0.03^{b}$ \\
\hline Total platelet count (cells/mm³) & $85,000(52,750-113,250)$ & $91,500(48,250-138,000)$ & 0.65 \\
\hline Hemoglobin (gm/dl) & $13.9 \pm 1.9$ & $13.3 \pm 1.5$ & 0.10 \\
\hline Hematocrit (\%) & $41.4 \pm 5.4$ & $40 \pm 4.3$ & 0.20 \\
\hline Random blood sugar (mg/dl) & 109 (99-132) & $111(102-122)$ & 0.85 \\
\hline Total bilirubin (mg/dl) & $1.3(0.8-2.1)$ & $1.6(0.8-2.4)$ & 0.87 \\
\hline Direct bilirubin (mg/dl) & $0.5(0.3-0.8)$ & $0.5(0.3-1.1)$ & 0.81 \\
\hline Serum aspartate aminotransferase (IU/L) & $37(25.5-58)$ & $28.5(19-45.8)$ & 0.05 \\
\hline Serum alanine aminotransferase (IU/L) & $41(24.5-69)$ & $26.5(18-44.3)$ & $0.01^{b}$ \\
\hline Serum alkaline phosphatase (U/L) & $88(69.5-114.5)$ & $85.5(73.5-106.8)$ & 0.97 \\
\hline Serum urea (mg/dl) & $24(17-31.3)$ & $25(21-33.5)$ & 0.44 \\
\hline Serum creatinine (mg/dl) & $1.0 \pm 0.3$ & $1.0 \pm 0.4$ & 0.92 \\
\hline Parasite index (parasites $/ \mu$ l blood) ${ }^{c}$ & $1,232(936-1,623)$ & $1,599(1,048-2,440)$ & 0.36 \\
\hline
\end{tabular}

andependent sample $t$-test or Mann Whitney $U$ test was performed.

${ }^{\mathrm{b} P}$-value less than 0.05 showing statistically significant differences.

${ }^{\circ}$ Geometric mean with $95 \%$ confidence interval. 
Table 3. Comparison of demographic and clinical variables during initial presentation and first recurrence among recurrence group

\begin{tabular}{|c|c|c|c|}
\hline Variables & At initial presentation $(\mathrm{N}=28)(\%)$ & At first recurrence $(\mathrm{N}=28)(\%)$ & $P$-value ${ }^{a}$ \\
\hline $\begin{array}{l}\text { Point-of-care } \\
\text { Ambulatory } \\
\text { Inpatient }\end{array}$ & $\begin{array}{l}16(57.1) \\
12(42.9)\end{array}$ & $\begin{array}{r}25(89.3) \\
3(10.7)\end{array}$ & $0.02^{b}$ \\
\hline $\begin{array}{l}\text { History } \\
\text { Headache } \\
\text { Cough } \\
\text { Vomiting } \\
\text { Diarrhea }\end{array}$ & $\begin{array}{c}24(85.7) \\
13(46.4) \\
13(46.4) \\
1(3.6)\end{array}$ & $\begin{aligned} 14 & (50.0) \\
7 & (25.0) \\
5 & (17.9) \\
2 & (7.1)\end{aligned}$ & $\begin{array}{l}0.01^{b} \\
0.11 \\
0.04^{b} \\
1.00\end{array}$ \\
\hline $\begin{array}{l}\mathrm{nPCR} \text { diagnosis } \\
P \text {. vivax mono-infection } \\
\text { Mixed } P \text {. vivax and } P \text {. falciparum }\end{array}$ & $\begin{array}{r}22(78.6) \\
6(21.4)\end{array}$ & $\begin{array}{r}22(78.6) \\
5(17.9)^{c}\end{array}$ & 1.00 \\
\hline Severe malaria $^{d}$ & $3(10.7)$ & 0 & 0.24 \\
\hline $\begin{array}{l}\text { Antimalarial treatment } \\
\text { CQ-PQ } \\
\text { ACT-PQ }\end{array}$ & $\begin{array}{r}22(78.6) \\
6(21.4)\end{array}$ & $\begin{array}{c}27(96.4) \\
1(3.6)\end{array}$ & 0.07 \\
\hline
\end{tabular}

McNemar chi-square test.

${ }^{b} P$-value less than 0.05 showing statistically significant differences.

'Blood sample could not be obtained at first recurrence for DNA extraction.

'As per the WHO's guideline for management of severe malaria in the year 2012.

Table 4. Comparison of laboratory variables during initial presentation and first recurrence among recurrence group

\begin{tabular}{|c|c|c|c|}
\hline \multirow{2}{*}{ Variables } & At initial presentation $(\mathrm{N}=28)$ & At first recurrence $(\mathrm{N}=28)$ & \multirow{2}{*}{$P$-value ${ }^{a}$} \\
\hline & Mean \pm SD or median (IQR) & Mean \pm SD or median (IQR) & \\
\hline Total leucocyte count (cells/mm³) & $5,907 \pm 1,905$ & $6,250 \pm 1,417$ & 0.36 \\
\hline Total platelet count (cells/mm³) & $91,500(48,250-138,000)$ & $113,000(96,250-142,750)$ & 0.27 \\
\hline Hemoglobin (gm/dl) & $13.3 \pm 1.5$ & $13.8 \pm 1.7$ & $0.03^{b}$ \\
\hline Hematocrit (\%) & $40 \pm 4.3$ & $42.2 \pm 5.1$ & $0.03^{b}$ \\
\hline Random blood sugar (mg/dl) & $111(102-122)$ & $111(93.5-140.5)$ & 0.40 \\
\hline Total bilirubin (mg/dl) & $1.6(0.8-2.4)$ & $1.1(0.6-1.5)$ & 0.07 \\
\hline Direct bilirubin (mg/dl) & $0.5(0.3-1.1)$ & $0.4(0.2-0.6)$ & 0.10 \\
\hline Serum aspartate aminotransferase (IU/L) & $28.5(19-45.8)$ & $22(19.8-30.3)$ & 0.23 \\
\hline Serum alanine aminotransferase (IU/L) & $26.5(18-44.3)$ & $19(13.8-34.3)$ & 0.26 \\
\hline Serum alkaline phosphatase (U/L) & $85.5(73.5-106.8)$ & $72(57-87)$ & 0.10 \\
\hline Serum urea (mg/dl) & $25(21-33.5)$ & $23.5(18.3-26.8)$ & 0.35 \\
\hline Serum creatinine (mg/dl) & $1.0 \pm 0.4$ & $0.9 \pm 0.1$ & 0.22 \\
\hline Parasite index (parasites $/ \mu$ l blood) ${ }^{c}$ & $1,599(1,048-2,440)$ & $2,079(1,211-3,569)$ & 0.39 \\
\hline
\end{tabular}

apaired $t$-test or Willcoxon sign rank test was performed.

${ }^{\mathrm{b}} \mathrm{P}$-value less than 0.05 shows statistically significant difference and shown in bold font.

${ }^{\circ}$ Geometric mean with 95\% confidence interval.

except hemoglobin and hematocrit showed a significant difference between initial presentation and first recurrence (Table 4).

Participants with history of $P$. vivax malaria did recur in significantly higher $(P=0.02)$ proportion and had a higher HR for recurrences of 2.62 (95\% CI; 1.24-5.54) $(P=0.012$; Fig. $3 \mathrm{~B})$. There was no significant difference $(P=0.10)$ in the duration of past malaria between recurrence and no-recurrence groups. The proportion of severe malaria cases did not vary $(P=1.00)$ between recurrence and no-recurrence groups. Of 28 cases in recurrence group, 3 (10.7\%) had severe malaria initially; however, none developed severe malaria during sub- sequent recurrences.

Fig. 4 is a flow diagram depicting results of nPCR test in recurrence cohort at different recurrence stages. At all recurrence stages, including R0, i.e., at initial presentation, while light microscopy test showed mono-infection with $P$. vivax, nPCR test did reveal subpatent mixed infections. Adjoining lines do indicate nPCR test results with the corresponding group of participants across all recurrence stages. At R0, of all 28 P. vivax mono-infections as diagnosed by light microscopy, only 22 did have P. vivax mono-infections as confirmed by the nPCR test. At R1, of 22 nPCR proven P. vivax mono-infections, only 16 

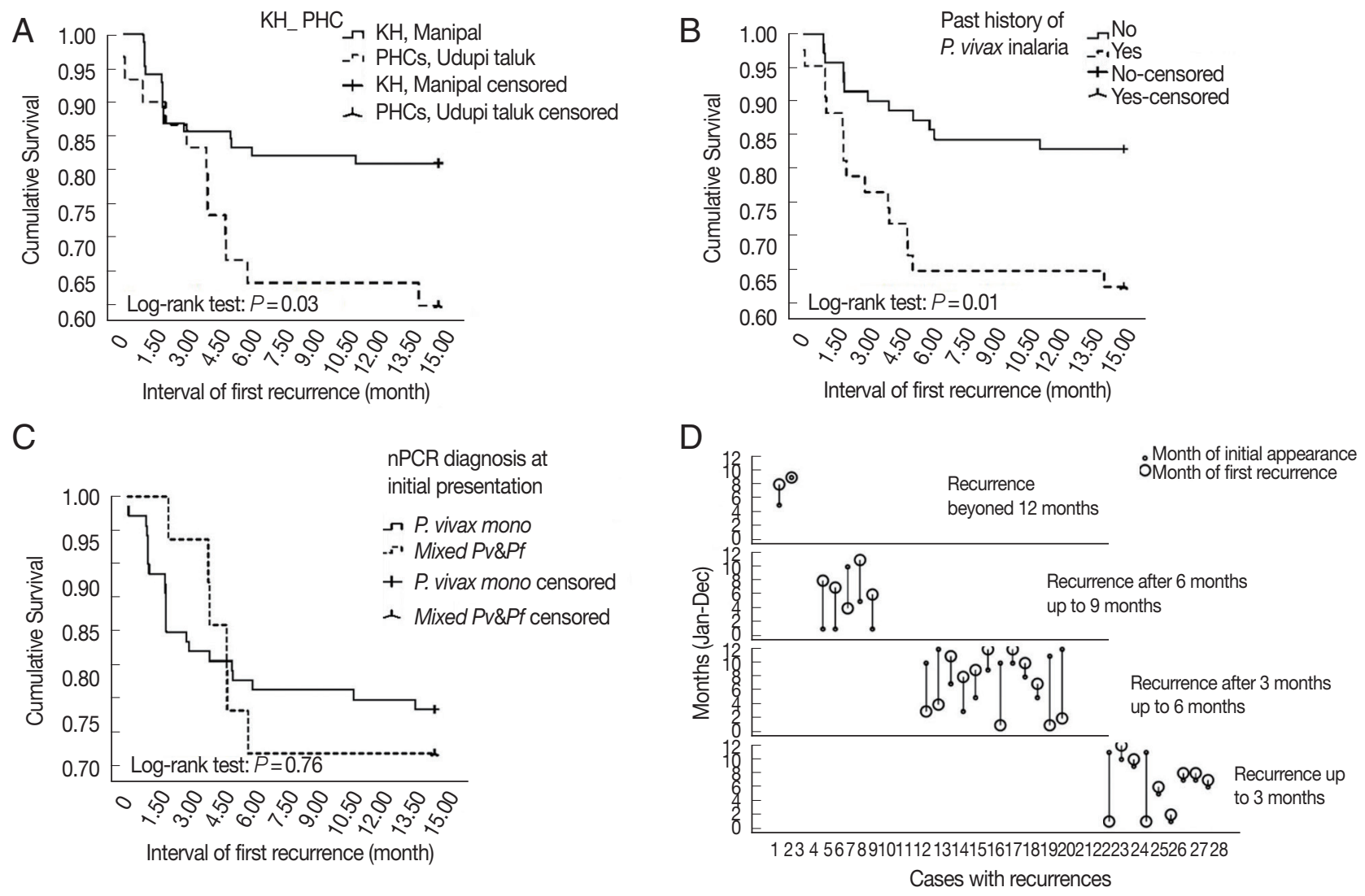

Fig. 3. (A) Kaplan-Meier survival plot showing recurrence probability up to 15 months among participants from KH, Manipal and PHCs, Udupi taluk. (B) Kaplan-Meier survival plot showing recurrence probability up to 15 months among participants with and without history of $P$. vivax malaria. (C) Kaplan-Meier survival plot showing recurrence probability up to 15 months among participants diagnosed with $P$. vivax mono-infection and mixed malaria by nested PCR test at initial presentation. (D) Drop-line graph depicting relation between months of initial appearance and first recurrence stratified by duration of recurrences.

were found to have nPCR proven mono-infections. Hence, the nPCR proven $P$. vivax mono-infection recurrence rate was at least $72.7 \%$ (16/22) at first recurrence. All mixed infections at R0 did have nPCR proven mono-infection at R1. Revelation of nPCR proven mixed infection at subsequent recurrence indicated possible new infection with $P$. falciparum and/or simultaneous inoculation of new P. vivax strain as well. There was no significant $(P=0.76)$ difference in the cumulative recurrence hazard function between nPCR diagnosed $P$. vivax mono-infection and mixed malaria groups at initial presentation (Fig. 3C).

In recurrence cohort, the initial infection occurred across the year except in the month of February and April. Subsequently, their first recurrence was noted throughout the year except in the month of May. There was no distinct seasonality noted for recurrences (Fig. 3D).

\section{DISCUSSION}

This study was conducted to assess the anti-recurrence efficacy of PQ regimen $(0.25 \mathrm{mg} / \mathrm{kg}$ body weight daily over 14 days) in $P$. vivax malaria. During acute $P$. vivax illness, patients were treated with CQ-PQ combined regimen as mentioned in the methods section. Convalescent and microscopically aparasitemic voluntary participants after acute $P$. vivax malaria on day 28 were re-enrolled into the 15 months' long follow-up study.

Male predominance in this study is a reflection of the gender ratio in cohort of preceding CQ-PQ combined therapeutic trials $[13,14]$. However, the male preponderance in malaria have also been reported previously [19-21] from the current study setting.

Duration of follow-up considerably influences the observed recurrence proportion in studies involving therapeutic assess- 


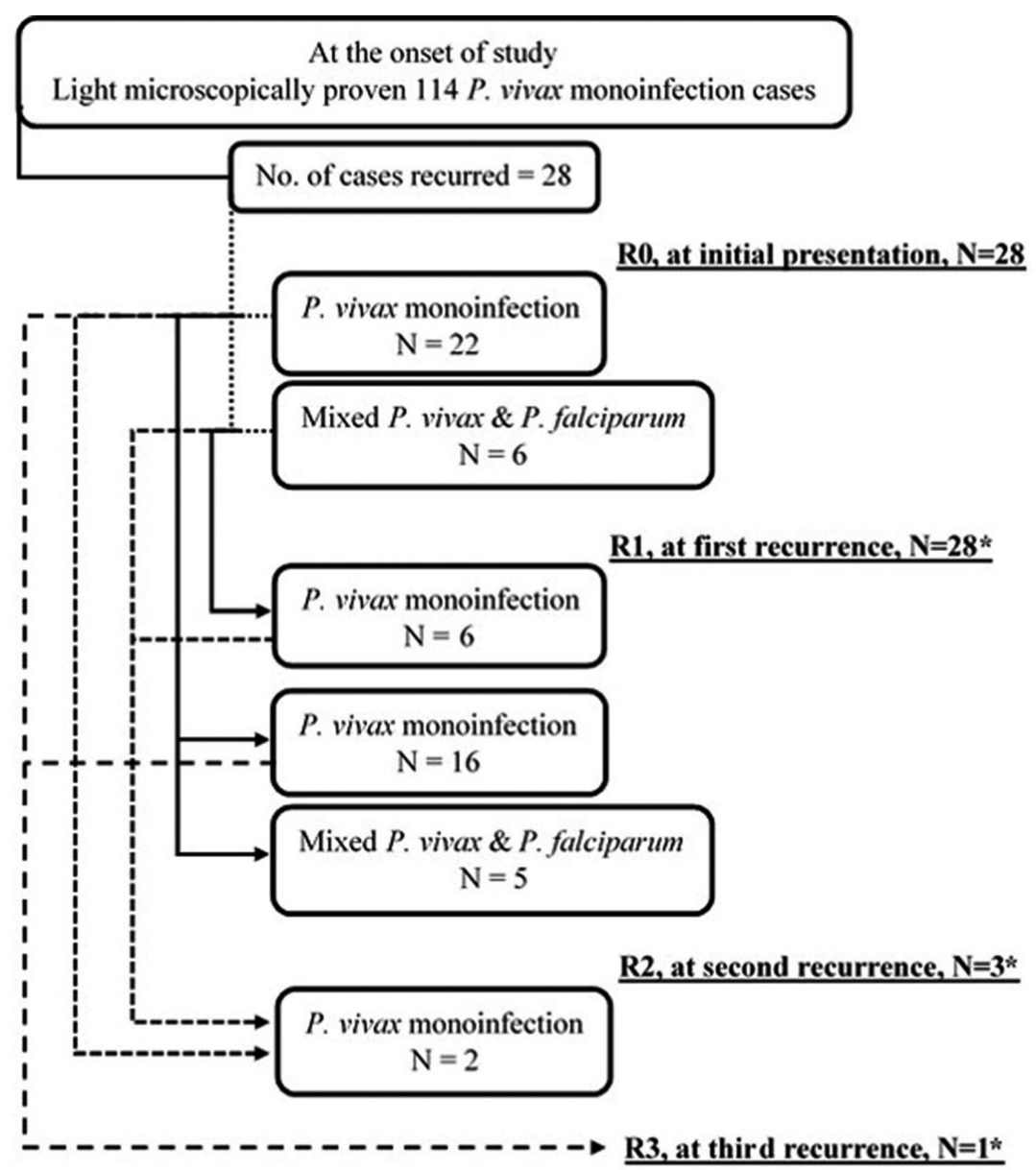

*Blood sample for one case each at $R 1, R 2$ and $R 3$ recurrences were not obtained for DNA extraction and NPCR test.

Fig. 4. Flow diagram depicting results of nested PCR test in recurrence cohort at different recurrence stages.

ment of PQ in P. vivax malaria [2]. Differences in the length of follow-up, antimalarial regimen, PQ dosage, and participants' age in studies [2] pose a challenge of heterogeneity to the comparison of their observed recurrence proportions. In unsupervised therapeutic studies with CQ, 1,500 mg over 3 days and PQ 210 mg over 14 days, P. vivax recurrence was reported to have varied from $8.1 \%$ across 6 months to $38.0 \%$ across 15 months [2]. The recurrence proportion $(24.6 \%, 28 / 114)$ over 16 months as observed in this study was less than the proportion $(38.1 \%, 16 / 42)$ observed by Kim et al. [10] over 15 months in Kolkata, India for the same CQ-PQ regimen as described in this study.

Similar to the findings of this study (Table 3), previously Adak et al. [8] did observe comparatively milder disease manifestation in recurring $P$. vivax cases than their initial presentation. It seems plausible that severity of disease does not deter- mine the subsequent recurrences of $P$. vivax, rather subsequent episodes are likely to be clinically milder due to acquired immunity [22]. However, development of acquired immunity for $P$. vivax and its impact on the course of clinical illness in this low transmission intensity study area remains elusive and needs a thorough validation. In this study, history of $P$. vivax malaria has surfaced as a sole independent predictor of subsequent recurrences. Of note, an empiric evidence of intact CQPQ therapeutic efficacy for subpatent $P$. falciparum infection has surfaced in this study. All 6 participants having nPCR proven mixed infections at R0, did show nPCR proven $P$. vivax mono-infection at R1 (Fig. 4). This indicates that CQ-PQ combined regimen at R0 did effectively cleared subpatent $P$. falciparum parasitemia along with patent $P$. vivax parasitemia. However, this empiric evidence needs to be verified further. Conversely, nPCR proven mixed infections (patent $P$. vivax and 
subpatent $P$. falciparum) cases from KH, Manipal and PHCs, Udupi taluk did have adequate clinical and parasitological responses to the CQ-PQ combined regimen $[13,14]$.

Rationally long follow-up duration (16 months), robust study design, statistical analyses, microscopic and nPCR confirmation of $P$. vivax mono-infection render the outcomes of this study valid and comparable with other similar studies. To the best of our knowledge, this is the first report of its kind from the study region, i.e., Udupi district.

This study has certain limitations as well. Firstly, we cannot rule out the possibility of intermittent self-limiting asymptomatic recurrences, thus it remains plausible that there would have been even higher rate of recurrences than observed in this study. Because the study setting is malaria endemic and recurrences occurred across the year, proportion of recurrence having 'reinfection' needs to be determined. As about $50 \%$ of recurrences did occur during regional rainy season between the months of June to October (Fig. 3D), a substantial proportion could be 'reinfection', and its confirmation requires further genetic investigations $[2,10]$. Also, a higher cumulative recurrence propensity (Fig. 3A) in P. vivax cohort of PHCs' than $\mathrm{KH}$, Manipal could be due to 'reinfection' rather than 'relapse', and further genetic analyses are required for confirmation $[2,10]$. This notion of reinfection arose by the circumstance wherein the majority $(83.3 \%, 10 / 12)$ of the recurrence participants of PHCs were from Hiriadka PHC, and all of them were workers of various mega-construction sites around which local malaria transmission is intensely confined. Conversely, differences in proportion of participants complying with the PQ regimen and underlying CYP2D6 metabolizer phenotypes [2] between PHCs and $\mathrm{KH}$ cohort could also explain the observed differences in cumulative recurrence between the cohorts of 2 settings. As the CQ-PQ regimen was unsupervised, non-compliance with 14 days long PQ could not be ruled out among participants. Further, like findings of Kim et al. [10] from Kolkata, India both short and long interval latency $P$. vivax phenotypes have been found in the present study (Fig. 3D). Howev$\mathrm{er}$, further genetic analyses are required to describe the individual parasite strains and their temporal association with recurrence. Also, it would be interesting to study the clonal variations among $P$. vivax genotypes in both nPCR proven mono-infection and mixed cases through all recurrence stages.

In Udupi district, PQ dose of $0.25 \mathrm{mg} / \mathrm{kg} /$ day over 14 days seems inadequate to prevent recurrences in substantial proportion of $P$. vivax malaria. Patients with a history of $P$. vivax malaria are at high risk of recurrences. Further, molecular investi- gations are required to determine the true rate of 'relapse' and its determinants.

\section{ACKNOWLEDGMENTS}

This work was supported by an extramural research grant (no. AMR/46/2011-ECD-I) and a Senior Research Fellowship Grant (no. 80/93/2015-ECD-I) from the Indian Council of Medical Research, New Delhi, India. The funding council had no role in study design, data collection and analysis, decision to publish, or preparation of the manuscript. Authors are immensely grateful to all the participants of this study. Much of the field work carried out by Mr. Satish Kitta Madivala and Mr. Prashantha Prabhu, Laboratory Assistants, is thankfully acknowledged.

\section{CONFLICT Of INTEREST}

We have no conflict of interest related to this work.

\section{REFERENCES}

1. WHO. World Malaria Report 2013. Geneva, Switzerland. World Health Organization. 2013.

2. Rishikesh K, Saravu K. Primaquine treatment and relapse in Plasmodium vivax malaria. Pathog Glob Health 2016; 110: 1-8.

3. White NJ. Determinants of relapse periodicity in Plasmodium vivax malaria. Malar J 2011; 10: 297.

4. WHO. World Malaria Report 2014. Geneva, Switzerland. World Health Organization. 2014.

5. Directorate of National Vector Borne Disease Control Programme. National Drug Policy on Malaria. 22-Shamnath Marg, Delhi-110054: Directorate General of Health Services: Ministry of Health and Family Welfare. 2013.

6. Srivastava HC, Sharma SK, Bhatt RM, Sharma VP. Studies on Plasmodium vivax relapse pattern in Kheda district, Gujarat. Indian J Malariol 1996; 33: 173-179.

7. Adak T, Sharma VP, Orlov VS. Studies on the Plasmodium vivax relapse pattern in Delhi, India. Am J Trop Med Hyg 1998; 59: 175-179.

8. Adak T, Valecha N, Sharma VP. Plasmodium vivax polymorphism in a clinical drug trial. Clin Diagn Lab Immunol 2001; 8: 891894.

9. Gogtay NJ, Desai S, Kadam VS, Kamtekar KD, Dalvi SS, Kshirsagar NA. Relapse pattern of Plasmodium vivax in Mumbai: a study of 283 cases of vivax malaria. J Assoc Physicians India 2000; 48: 1085-1086.

10. Kim JR, Nandy A, Maji AK, Addy M, Dondorp AM, Day NP, Pukrittayakamee S, White NJ, Imwong M. Genotyping of Plas- 
modium vivax reveals both short and long latency relapse patterns in Kolkata. PLoS One 2012; 7: e39645.

11. Gogtay N, Desai S, Kamtekar KD, Kadam VS, Dalvi SS, Kshirsagar NA. Efficacies of 5-and-14-day primaquine regimens in the prevention of relapses in Plasmodium vivax infections. Ann Trop Med Parasitol 1999; 93: 809-812.

12. Sinha S, Dua VK, Sharma VP. Efficacy of 5 day radical treatment of primaquine in Plasmodium vivax cases at the BHEL Industrial Complex, Hardwar (UP). Indian J Malariol 1989; 26: 83-86.

13. Rishikesh K, Kamath A, Hande MH, Vidyasagar S, Acharya RV, Acharya V, Belle J, Shastry AB, Saravu K. Therapeutic assessment of chloroquine-primaquine combined regimen in adult cohort of Plasmodium vivax malaria from a tertiary care hospital in southwestern India. Malar J 2015; 14: 310.

14. Saravu K, Kumar R, Ashok H, Kundapura P, Kamath V, Kamath A, Mukhopadhyay $\mathrm{C}$. Therapeutic assessment of chloroquine-primaquine combined regimen in adult cohort of Plasmodium vivax malaria from primary care centres in southwestern India. PLoS One 2016; 11: e0157666.

15. World Health Organization. Point-of-care G6PD testing to support safe use of primaquine for the treatment of vivax malaria. WHO Evidence Review Group meeting report. In Malaria Policy Advisory Committee Meeting. Geneva, Switzerland. WHO/UNAIDS Building. 2015.

16. Rishikesh K, Madivala SK, Prabhu P, Kamath A, Ashok H, Vidyasagar S, Shastry AB, Saravu K. Surmised total leucocyte counts miscalculate the parasite index of Plasmodium vivax ma- laria patients of tertiary and primary care settings in South-Western India. Malar J 2015; 14: 163.

17. World Health Organization. A practical handbook--Management of severe malaria. 3rd ed. Geneva, Switzerland. World Health Organization. 2012.

18. Snounou G, Viriyakosol S, Zhu XP, Jarra W, Pinheiro L, do Rosario VE, Thaithong S, Brown $\mathrm{KN}$. High sensitivity of detection of human malaria parasites by the use of nested polymerase chain reaction. Mol Biochem Parasitol 1993; 61: 315-320.

19. Saravu K, Rishikesh K, Parikh CR. Risk factors and outcomes stratified by severity of acute kidney injury in malaria. PLoS One 2014; 9: e90419.

20. Saravu K, Rishikesh K, Kamath A, Shastry AB. Severity in Plasmodium vivax malaria claiming global vigilance and exploration-a tertiary care centre-based cohort study. Malar J 2014; 13: 304.

21. Saravu K, Rishikesh K, Kamath A. Determinants of mortality, intensive care requirement and prolonged hospitalization in malaria-a tertiary care hospital based cohort study from SouthWestern India. Malar J 2014; 13: 370.

22. Doolan DL, Dobano C, Baird JK. Acquired immunity to malaria. Clin Microbiol Rev 2009; 22: 13-36.

23. Rishikesh K, Kamath A, Hande MH, Vidyasagar S, Acharya RV, Acharya V, Belle J, Shastry AB, Saravu K. Therapeutic assessment of chloroquine-primaquine combined regimen in adult cohort of Plasmodium vivax malaria from a tertiary care hospital in southwestern India. Malar J 2015; 14: 310. 